\title{
THE NUMBERS OF CHIRAL AND ACHIRAL ALKANES AND MONOSUBSTITUTED ALKANES
}

\author{
R. W. ROBINSON ${ }^{a, b . *}$ \\ Department of Mathematics, University of Newcastle, New South Wales, 2308, Australia \\ F. HARARY ${ }^{a}$ \\ Department of Mathematics, University of Michigan, Ann Arbor, MI 48104, U.S.A.
}

and

A. T. Balaban

Institute of Atomic Physics, P.O. Box 5206, Bucharest, Romania

(Received in UK 15 August 1974; Accepted for publication 2 September 1975)

\begin{abstract}
Whereas the theory for the enumeration of the optical isomers of the alkyl radicals and the alkanes has long been understood, this is not the case for the corresponding archiral isomers. We present for the first time recurrence formulae for counting the number of archiral isomers of the alkyl radicals and the alkanes. For chiral and archiral alkanes and monosubstituted alkanes, numerical results up to $C_{14}$ are tabulated.

After presenting the history of the problem and the necessary definitions, we proceed to derive functional equations on the various generating functions, which readily yield the more explicit recurrence formulae useful for numerical calculations. In the process, we first re-derive Pólya's expression for planted steric trees using his classical enumeration theorem. This result is then extended to the enumeration of free steric trees using the now standard tree-counting method due to Otter and known as a dissimilarity characteristic equation.

By definition, a steric tree is a quartic tree (all points having degree 1 or 4) in which the four neighbors of every carbon point are given a tetrahedral configuration. Building on the methods of the first two authors for counting chiral and achiral trees in the plane, we obtain the formula for counting achiral steric trees, thus settling a problem first enunciated by van't Hoff and Le Bel in 1874 .
\end{abstract}

\section{DEFINITIONS AND HISTORY}

1.1 Quartic trees. A tree $\mathrm{T}$ is a graph (consisting of a finite collection of points, some joined by lines) which is connected and contains no cycles. The degree of a point is the number of other points to which it is adjacent. In a quartic tree, every point has degree 4 or 1 . There is a direct correspondence between quartic trees and structural formulas for the acyclic saturated hydrocarbons $\mathrm{C}_{n} \mathrm{H}_{2 n+2}$; i.e. the methane series. Each point of degree 4 in a quartic tree can be regarded as representing a carbon atom, while the points of degree 1 stand for hydrogen atoms and each line joining two points represents a single covalent bond.

Cayley ${ }^{1.2}$ first introduced quartic trees, and enumerated them up to 11 points of degree 4. Henze and Blair ${ }^{3,4}$ presented extensive numerical results, which were supplemented by those of Perry. ${ }^{5}$ Pólya ${ }^{6}$ in his classic paper included a derivation of counting formulas for quartic trees and a detailed asymptotic analysis. Otter' put the counting results entirely in terms of generating functions and gave more refined estimates of the constants in the asymptotic expansions.

For the sake of clarity in the present context we call a point of degree 4 of a quartic tree a carbon point, and call a line between two carbon points a carbon line. The order $n$ of a quartic tree is the number of carbon points it

${ }^{a}$ Research supported in part by Grant 73-2502 from the U.S. Air Force Office of Scientific Research.

'The authors thank the referee for making many helpful suggestions concerning the presentation of these results. contains. In this and some other respects, we shall not be following the standard terminology of graph theory. For detailed definitions and results in graph theory and graphical enumeration two recent books may be consulted. ${ }^{8.9}$ Extensive expositions of the chemical applications of graph theory can be found in the review papers of Rouvray. ${ }^{10}$ An account of recent enumeration results on structural isomers of the acyclic hydrocarbons is given by Read."

The points of degree 1 in a quartic tree are called endpoints. A quartic tree with a distinguished point is called a rooted carbon tree, and the distinguished point is called the root. Ordinary quartic trees are called free whenever it is desirable to emphasize that they are not rooted. A quartic tree in which the root point is an endpoint is called planted; if the root point is a carbon point, we shall call the tree carbon-rooted. Planted quartic trees correspond in a natural way to structural formulas for alkyl radicals or monosubstituted alkanes, just as free quartic trees correspond to alkanes, except that the root point does not represent a hydrogen atom, but the radical site (assumed pyramidal) or the substituent. It will be convenient to include in the class of planted quartic trees the trivial planted tree, consisting of a single isolated root point. This corresponds to the hydrogen atom $\mathrm{H}$, which will be considered as the smallest alkyl radical with $n=0$.

Quartic trees are deficient from a chemical point of view, in that no account is taken of the geometry of the molecules which they represent. The notion of isomorphism for quartic trees is strictly topological (what Pólya 
called structure-isomorphism) and corresponds to constitutional isomerism. Consequently the number of quartic trees is smaller than the number of actual chemical species (alkanes), because a particular quartic tree may correspond to several distinct alkanes, having the same structural formula but differing sterically (stereoisomers, in this case optical isomers).

Let $u_{n}$ and $v_{n}$ be the numbers of different planted and free quartic trees of order $n$. Then $u_{n}$ is the number of distinct structural isomers of the alkyl radicals $\mathrm{C}_{n} \mathrm{H}_{2 n+1}$, and $v_{n}$ is the number of distinct structural isomers of the alkanes $\mathrm{C}_{n} \mathrm{H}_{2 n+2}$. These and subsequent notations are summarized in Table 1 . The numbers $u_{n}$ and $v_{n}$ will not play any part in the sequel, but some numerical values will be included in Tables 2 and 3 for the purposes of comparison ${ }^{\dagger}$.

1.2 Steric trees. A steric tree is a quartic tree in which the four neighbors of every carbon point are given a tetrahedral configuration, the chemical term for a three-dimensional orientation. An isomorphism between steric trees is then a topological isomorphism in which the configurations of these neighborhoods are preserved.

Assigning a configuration to four points $\mathrm{P}_{1}, \mathrm{P}_{2}, \mathrm{P}_{3}, \mathrm{P}_{4}$, is equivalent to assigning these points to the vertices of a regular tetrahedron. In Fig. 1 the two possible configurations of the quadruple $P_{1}, P_{2}, P_{3}, P_{4}$ are represented in this manner.
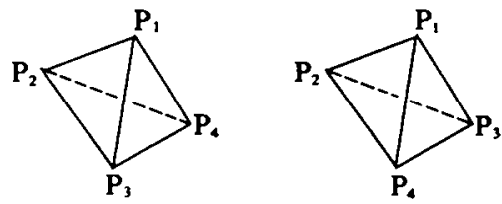

Fig. 1. The configurations (tri-dimensional orientations) of four points.

From the chemical point of view, we visualize the carbon atom at the center of the tetrahedron, with its four neighbours at the vertices. Any rearrangement of the neighbors is equivalent by a rotation to either the original configuration or its mirror image. Taking the mirror image is equivalent to reversing the configuration.

Let $s_{n}$ and $f_{n}$ be the numbers of different planted and free steric trees of order $n$. Values of $s_{n}$ and $f_{n}$ were calculated by laborious recursive procedures by Blair and Henze. ${ }^{16.17}$ Formulas for $s_{n}$ and $f_{n}$ were first given by Pólya ${ }^{6}$ who also derived asymptotic formulas containing constants which were not closely specified. Pólya's term for isomorphism of steric trees was "stereoisomorphism". Recently Read" ${ }^{18}$ used Pólya's counting theorem for compiling tables of numerical values which include $s_{n}$ and $f_{n}$, and various generalizations corresponding to unsaturated hydrocarbons.

In the obvious way steric trees can be thought of as representing optical isomers of the alkanes. Thus $s_{n}$ and $f_{n}$ count, respectively, the number of distinct optical isomers of the alkyl radicals or monosubstituted alkanes, and the

tThe referee pointed out that 1974 (when this paper was accepted for publication) represents an interesting double centenary: one hundred years since Cayley' introduced the numbers $u_{n}$ and $v_{n}$, and also one hundred years since the realization by van't Hoff ${ }^{12}$ and Le Bel ${ }^{13}$ of the tetrahedral valency of the carbon atom. The latter anniversary was commemorated in two special numbers of Tetrahedron; see Robinson ${ }^{14}$ and Riddell and Robinson. ${ }^{15}$
Table 1. Notations for numbers of trees of order $n$, of various types

\begin{tabular}{ccc}
\hline Troe & planted & free \\
\hline Quartic & $u_{n}$ & $v_{n}$ \\
Steric & $s_{n}$ & $f_{n}$ \\
Chiral (steric) & $g_{n}$ & $e_{n}$ \\
Achiral (steric) & $p_{n}$ & $t_{n}$ \\
\hline
\end{tabular}

Table 2. The numbers of planted trees or monosubstituted alkanes of various sorts

\begin{tabular}{rrrrr}
$n$ & $\begin{array}{c}\text { Achiral } \\
P_{n}\end{array}$ & $\begin{array}{c}\text { chiral } \\
\varepsilon_{n}\end{array}$ & $\begin{array}{c}\text { Steric } \\
s_{n}\end{array}$ & $\begin{array}{c}\text { Quartic } \\
u_{n}\end{array}$ \\
\hline 0 & 1 & 0 & 1 & 1 \\
1 & 1 & 0 & 1 & 1 \\
2 & 1 & 0 & 1 & 1 \\
3 & 2 & 0 & 2 & 2 \\
4 & 3 & 2 & 5 & 4 \\
5 & 5 & 6 & 11 & 8 \\
6 & 3 & 20 & 28 & 17 \\
7 & 14 & 60 & 24 & 39 \\
8 & 23 & 176 & 199 & 89 \\
9 & 41 & 510 & 551 & 211 \\
10 & 69 & 1,484 & 1,553 & 507 \\
11 & 122 & 4,314 & 4,436 & 1,238 \\
12 & 208 & 12,624 & 12,832 & 3,057 \\
13 & 370 & 37,126 & 37,406 & 2,639 \\
14 & 636 & 109,864 & 110,500 & 19,241 \\
\hline
\end{tabular}

Table 3. The numbers of free trees or alkanes of various sorts

\begin{tabular}{ccccc}
\hline$n$ & $\begin{array}{c}\text { Achiral } \\
t_{n}\end{array}$ & $\begin{array}{c}\text { Chiral } \\
\text { n }_{n}\end{array}$ & $\begin{array}{c}\text { Steric } \\
F_{n}\end{array}$ & $\begin{array}{c}\text { Quartic } \\
v_{n}\end{array}$ \\
\hline 1 & 1 & 0 & 1 & 1 \\
2 & 1 & 0 & 1 & 1 \\
3 & 1 & 0 & 1 & 1 \\
4 & 2 & 0 & 2 & 2 \\
5 & 3 & 0 & 3 & 3 \\
6 & 5 & 0 & 5 & 5 \\
7 & 7 & 4 & 11 & 9 \\
8 & 14 & 10 & 24 & 18 \\
9 & 21 & 34 & 55 & 35 \\
10 & 40 & 96 & 136 & 75 \\
11 & 61 & 284 & 345 & 159 \\
12 & 118 & 782 & 900 & 355 \\
13 & 196 & 4,226 & 2,412 & 802 \\
14 & 365 & 6,198 & 6,563 & 1,858 \\
\hline
\end{tabular}

alkanes, with exactly $n$ carbon atoms. However, there are two respects in which the correspondence between steric trees and optical isomers may not be perfectly satisfactory from the chemical standpoint. First, conformational isomerism resulting from possible steric hindrance is ignored. That is, one must assume free rotation about all single carbon-carbon bonds. Second, not all geometrical arrangements of atoms corresponding to the various steric trees are equally feasible. Note that both of these difficulties arise because the additional structure of the steric tree relative to the quartic tree describes only the local three-dimensional structure about each carbon atom, and does not specify the structure globally.

1.3 Chiral and achiral trees. The reverse of a steric tree is obtained by altering the configuration of the neighbors of every carbon point (Fig. 1). In the molecule which the steric tree represents, the reverse corresponds to taking 
the mirror-image. A steric tree is said to be chiral if it is distinct, as a steric tree, from its reverse. A chiral tree and its reverse are an enantiomeric pair; they represent a pair of corresponding enantiomers. A racemic mixture would have equal quantities of these corresponding enantiomers. Steric trees which are not chiral are called achiral. Therefore every steric tree is either chiral or achiral. Blair and Henze ${ }^{16.17}$ used the terms stereoisomorphic chiral and non-stereoisomorphic for achiral. Throughout the present paper, unless otherwise stated, chiral trees and achiral trees refer to steric trees, unlike the plane chiral and achiral trees enumerated previously by Harary and Robinson. ${ }^{19}$

The smallest chiral trees have order 7 , and correspond to the structural formulas 1 and 2 presented in Fig. 2. In each case, the tertiary carbon atom which is shown with its four bonds drawn as full lines has two possible configurations for its four neighbors, giving rise to a pair of enantiomers.<smiles>CCC(C)C(C)C</smiles>

1<smiles>CCCC(C)CC</smiles>

2

Fig. 2. The two racemic heptanes corresponding to the two smallest chiral trees.

For steric trees of order 8 , there are five structural isomers (3-7) which can give rise to chiral trees, presented in Fig. 3. Each of the first four leads to one pair of enantiomeric trees. The fifth one (7) leads to a pair of enantiomeric trees and to an achiral tree (internally compensated meso-form).<smiles>CCCCC(C)CC</smiles>

3<smiles>CCC(C)C(C)(C)C</smiles>

5<smiles>CCCC(C)C</smiles>

4<smiles>CCCC(C)C(C)C</smiles>

6<smiles>CCC(C)C(C)CC</smiles>

7

Fig. 3. The five octane isomers corresponding to the chiral trees of order 8.

The importance of chiral molecules in chemistry needs hardly be emphasized since proteins and glucides with their components are chiral. A striking example is that the molecules responsible for the scents spearmint and orange are an enantiomeric pair. ${ }^{20}$ It should be noted that the optical rotation of the chiral alkanes tested so far has been undetectably small." However, it may be possible to enhance the effect on admixture with a nematic liquid crystal: this would be converted into a cholesteric mesophase with detectable optical rotation, though neither the alkane nor the nematic mesophase is optically active. Alternatively, nuclear magnetic resonance techniques could be employed with the chiral heptane isomers: magnetic non-equivalence of diastereotopic $\mathrm{CH}_{2}$ (ethyl or $n$-propyl) or $\mathrm{CMe}_{2}$ (isopropyl) groups, or the use of chiral solvents, would be appropriate, even with the racemic heptanes.

Let $g_{n}$ and $e_{n}$ be the numbers of planted and free chiral trees of order $n$, and let $p_{n}$ and $t_{n}$ be the corresponding numbers of achiral trees. Clearly, $g_{n}=s_{n}-p_{n}$ and $e_{n}=f_{n}-t_{n}$, while $g_{n} / 2$ and $e_{n} / 2$ are the numbers of enantiomeric pairs of planted and free steric trees of order $n$. Thus $g_{n} / 2$ and $e_{n} / 2$ are the numbers of enantiomeric pairs of optical isomers of alkyl radicals and alkanes, respectively, with exactly $n$ carbon atoms, and $p_{n}$ and $t_{n}$ are the corresponding numbers of archiral forms.

The branches of a carbon-rooted quartic or steric tree are the four planted trees obtained by deleting the root point, which leaves four connected components. Each of these is made planted by supplying it with a new root point which is connected to the same point to which the original carbon root point was connected; or in the case of an empty subtree we form the trivial planted tree. A carbon point of a quartic tree is called asymmetric whenever the four branches at that point are all distinct. The carbon atoms which in Figs. 2 and 3 are drawn with all four bonds as full lines are precisely the ones which correspond to asymmetric points. Pólya ${ }^{6}$ counted quartic trees in which no carbon point is asymmetric. Unless a carbon point is asymmetric the two possible tetrahedral configurations which can be assigned to its neighbors are sterically equivalent. Thus the quartic trees counted by Pólya have just one steric version each, which is achiral. However, it is easy to see that such achiral trees are only a portion of the total; for example, they do not include the achiral tree which is associated with the fifth structural formula, 7, meso-form, of Fig. 3.

Two of the present authors ${ }^{19}$ explored the idea of chirality for planes trees: a plane tree is chiral if it is not plane-equivalent to its reflection about a line in the plane. In particular, they found the explicit formulas $\left(\begin{array}{c}n-2 \\ n / 2-1\end{array}\right)$ for the number of achiral plane trees of even order $n$, and $\frac{1}{2}\left(\begin{array}{c}n-1 \\ (n-1) / 2\end{array}\right)$ for the number of odd order. The number of planted achiral plane trees of order $n+1$ was found (surprisingly) to be the same. Some of the fundamental ideas of that paper ${ }^{19}$ are applied in the present one. It should be noted that in the case of plane trees no restrictions were made on the degrees of the points. But the main reason for the many differences in detail between this and the preceding paper is, of course, the passage from two dimensions to three.

For free trees we consider that the number of order 0 is 0 , that is, there must be at least one point of degree 4 representing a carbon atom; compare Harary and Read. ${ }^{22}$ However, we do count the trivial planted tree of order 0 , considering it in the steric case to be achiral.

The ordinary generating functions for the various types of trees listed in Table 1 are given parallel nomenclature 
using the same letters. Thus

$$
s(x)=\sum_{n=0}^{\infty} s_{n} x^{n}
$$

is the generating function for planted steric trees, while

$$
f(x)-\sum_{n=1}^{\infty} f_{n} x^{n}
$$

is that for free steric trees, and so on.

\section{COUNTING STERIC TREES}

2.1 Planted steric trees. First we establish the functional relation

$$
s(x)=1+\frac{x}{3}\left(s^{3}(x)+2 s\left(x^{3}\right)\right)
$$

which was first noted by Pólya, ${ }^{6}$ who proved it using his famous counting theorem, or Hauptsatz. We then derive a recurrence relation suitable for computing the numbers $s_{n}$.

Each non-trivial planted steric tree counted by $s(x)$ can be viewed as a function from the vertices of a regular tetrahedron to the four branches at the carbon point adjacent to the root point. The number of distinct functions of this sort is given by Pólya's Hauptsatz. ${ }^{6}$ We shall endeavour to outline the main features of the proof of the Hauptsatz, but for a full description the reader should consult the book.".

The first step in applying the Hauptsatz is to establish the permissible symmetry group on the vertices of the tetrahedron. One vertex is mapped to the trivial tree associated with the root point. The root point is preserved by every isomorphism of planted trees, so the corresponding vertex is always left fixed; for our purposes this vertex can be ignored. The other three vertices of the tetrahedron may be permuted by any rotation of the tetrahedron which leaves the root vertex fixed, that is just two $120^{\circ}$ rotations and the identity. The "cycle index" of this permutation group is that of the cyclic group $C_{3}$ of degree 3:

$$
\frac{1}{3}\left(a_{1}^{3}+2 a_{3}\right)
$$

Here $a_{1}$ and $a_{3}$ are part of the sequence $a_{1}, a_{2}, a_{3}, \ldots$ of independent commuting variables. The identity rotation is represented by $a_{1}{ }^{3}$ as it is the product of 3 cycles of length 1. Each of the $120^{\circ}$ rotations is represented by $a_{3}$ as it permutes the three vertices in a single cycle of length 3 . The cycle index is defined as the average of these terms over the whole permutation group.

Now, the non-trivial planted steric trees are in one-to-one correspondence with functions from the three free vertices of our tetrahedron into the set of all planted steric trees, provided that we consider any two of these functions as equivalent if we can get one get one from the other by applying one of the allowed permutations on the vertices. According to the Hauptsatz, the series for counting inequivalent functions of this sort is given by the expression

$$
\frac{1}{3}\left(s^{3}(x)+2 s\left(x^{3}\right)\right)
$$

The procedure is to substitute $s(x)$, the counting series for the range objects (into which the functions are mapping) for $a_{1}, s\left(x^{2}\right)$ for $a_{2}, s\left(x^{3}\right)$ for $a_{3}$, etc. in the cycle index for the permutation group which acts on the domain objects (from which the functions are mapping). In the resulting counting series, every carbon point is accounted for by a factor of $x$ except for the one adjacent to the root. Multiplying by $x$ to remedy this and adding 1 for the trivial planted tree, we obtain the right-hand side of (1).

Pólya's Hauptsatz is based on Burnside's Lemma, ${ }^{23}$ which states that the number of objects which are inequivalent under the action of a permutation group is the average (over the group) of the number of objects left fixed by each permutation. One applies this by regarding the permutations on the domain set (three vertices of a tetrahedron in the case just considered) as acting on the set of functions to be counted. To count inequivalent functions, then, one simply substitutes for each term in the cycle index representing a permutation the counting series for the functions which this permutation leaves fixed. This amounts to substituting $s(x)$ for $\left.a_{1}, s(x)^{2}\right)$ for $a_{2}$, and so on, in case $s(x)$ is the counting series for the range objects.

Upon differentiating (1) and then using (1) itself to simplify the result one obtains the differential equation

$$
x s^{\prime}(x)-s(x)+1=x\left(x s^{\prime}(x) s^{2}(x)+2 x^{3} s^{\prime}\left(x^{3}\right)\right)
$$

Let $q_{n}$ be the coefficient of $x^{n}$ in $s^{2}(x)$. Then $q_{n}$ is given by the relation

$$
q_{n}=s_{n / 2}^{2}+2 \sum_{k=n / 2} s_{k} s_{n-k}
$$

in which we take $s_{n i 2}$ to be 0 whenever $n$ is odd, i.e., whenever the subscript is not integral; this convention is assumed in the sequel to apply to any similar expressions.

Now equating coefficients of like powers of $x$ in (2) gives

$$
s_{n+1}=\frac{1}{n}\left\{\frac{2 n}{3} s_{n / 3}+\sum_{k=1}^{n} k s_{k} q_{n-k}\right\}
$$

for $n \geqslant 1$. Relations (3) and (4) with the initial values $s_{0}=s_{1}=1$ give a convenient way of computing successive values of $s_{n}$.

It should be observed that on introducing one substituent into an alkane, chirality may appear in this process; if the alkane was achiral, the monosubstituted alkane becomes chiral either when the substituent is introduced at a secondary carbon with asymmetric branches, e.g., 8-10 in Fig. 4, or when it causes two otherwise symmetric branches to become distinct, when they are attached to a carbon point whose two other branches are already distinct, e.g., 11-15. If the free steric tree (alkane) was chiral, conversion into a planted steric tree (monosubstituted alkane) conserves that chirality and may add a new chirality at the rooted point.

Figure 4 illustrates the smallest chiral planted trees, i.e., the single enantiomeric pair of chiral planted trees of order $4(8)$, and the three enantiomeric pairs of chiral planted trees of order $5(9-11)$. The remaining examples from Fig. 4 illustrate, in the case of planted steric trees of orders 6-9, the presence of more than one chiral vertex in one planted steric tree: 12-14 have two such vertices leading each to four chiral planted trees, 15 has three such vertices and leads to eight chiral planted trees, while 16 also 


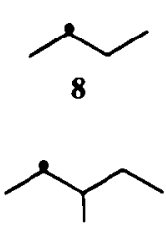

12
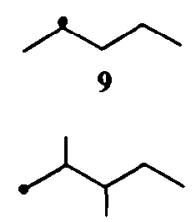

13

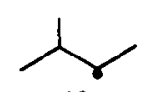

10

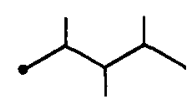

14

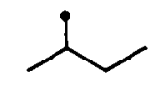

11

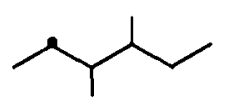

15

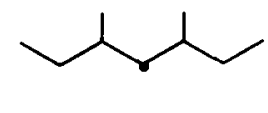

16

Fig. 4. Chiral planted trees (the dot corresponds to the carbon point bonded to the root point, or to the carbon atom bonded to the univalent substituent in the monosubstituted alkane).

has three such vertices and leads to only two chiral planted trees and two achiral planted trees.

All these different kinds of chirality in monosubstituted alkanes are taken into account in the figures presented in Table 2.

2.2 Free steric trees. In order to obtain $f(x)$ in terms of $s(x)$ we use a variant of the dissimilarity characteristic equation of Otter. ${ }^{7}$ Call two points of a steric tree $T$ similar if and only if some steric automorphism of $T$ maps one of them to the other. Let $p^{*}(T)$ be the number of dissimilar carbon points in $T$. Likewise, let $q^{*}(T)$ be the number of dissimilar carbon lines in $T$. Now, in case there is some steric automorphism of $T$ which interchanges two adjacent carbon points we let $s^{*}(T)=1$, and otherwise we let $s^{*}(T)=0$. Then the dissimilarity equation takes the form

$$
p^{*}(T)-q^{*}(T)+s^{*}(T)=1,
$$

which is true of every steric tree $T$.

The proof of (5) hinges on the observation that any steric tree $T$ satisfies either (i) or (ii);

(i) there is some point $v$ in $T$ which is left fixed by every steric automorphism of $T$, or

(ii) there is some line of $T$ which has its ends interchanged by some steric automorphism of $T$ and this line is left fixed by all automorphisms of $T$.

This can be shown by producing some topological invariant of the tree $T$ which turns out always to consist of a single point or else two adjacent points. One such invariant is the center of $T$, i.e., the subtree induced by those points of $T$ for which the maximum distance to another point of $T$ is minimized (see Ref. 8, p. 35)

Now, if $T$ satisfies (i), then $v$ is similar to no other point of $T$. Taking for each carbon line of $T$ the incident point which is further from $v$, we see that any two carbon lines are similar if and only if the corresponding points are similar. Thus each similarity class of carbon lines is matched by a similarity class of carbon points, with only the class of the unique carbon point which is nearest to $v$ not used in the matchings. Thus

$$
p^{*}(T)-q^{*}(T)=1
$$

which verifies (5) in case (i) since $s^{*}(T)=0$ in this case.

If $T$ satisfies (ii) we proceed from the central line, and find a perfect matching between the similarity classes of carbon lines and those of carbon points. Thus

$$
p^{*}(T)-q^{*}(T)=0
$$

But in this case $s^{*}(T)=1$, and so (5) is again verified and is now proved.

In Otter's statement of (5), similarity was taken with respect to the topologica' (structural or constitutional) automorphism group of the tree. However, it should be clear from our discussion above that for similarity taken with respect to any desired subgroup of the topological automorphism group, a dissimilarity characteristic equation analogous to (5) will hold true.

Let $n(T)$ be the order of tree $T$. We multiply both sides of (5) by $x^{n(T)}$ and sum over all steric trees to obtain

$$
\begin{aligned}
\sum p^{*}(T) x^{n(T)}-\sum q^{*}(T) x^{n(T)}+\sum s^{*}(T) x^{n(T)} & \\
& =\sum x^{n(T)}
\end{aligned}
$$

The right side of $(6)$ is just $f(x)$, as every steric tree is counted just once, with the appropriate weight. We now take the three terms of the left-hand side of (6) in order, and evaluate each in terms of $s(x)$.

The sum $\Sigma p^{*}(T) x^{n(T)}$ counts dissimilar carbon points in steric trees, or, what amounts to the same thing, it counts steric trees which are carbon-rooted. In order to apply Pólya's Hauptsatz to this situation, consider a carbon-rooted steric tree to be a function from the four vertices of a regular tetrahedron (representing the bonds of the carbon atom corresponding to the root point) to the four branches at the root. The permissible group of permutations on the vertices is the rotation group of the tetrahedron, consisting of the identity, 3 rotations of order 2 , and 8 rotations of order 3 . It is not hard to see, as in Pólya $^{6}$ or Hill, ${ }^{24}$ that the cycle index of this group is

$$
\frac{1}{12} a_{1}{ }^{4}+\frac{1}{4} a_{2}{ }^{2}+\frac{2}{3} a_{1} a_{3}
$$

Each branch at the root could be any of the planted steric trees, which are counted by the series $s(x)$. Thus, by Pólya's Hauptsatz we have

$$
\sum p^{*}(T) x^{n(T)}=\frac{x}{12} s^{4}(x)+\frac{x}{4} s^{2}\left(x^{2}\right)+\frac{2}{3} x s(x) s\left(x^{3}\right)
$$

since we must multiply by a factor of $x$ in order to include the root point in the count of carbon points.

To simplify this expression, note that the right-hand side may be arranged in the form

$$
\frac{1}{4} s(x)\left(\frac{x}{3} s^{3}(x)+\frac{2}{3} x s\left(x^{3}\right)\right)+\frac{x}{4} s^{2}\left(x^{2}\right)+\frac{x}{2} s(x) s\left(x^{3}\right) .
$$

The first product may be simplified using (1), giving for the first term of (6):

$$
\begin{aligned}
\sum p^{*}(T) x^{n(T)}=\frac{1}{4} s^{2}(x)-\frac{1}{4} s(x) & +\frac{x}{4} s^{2}\left(x^{2}\right) \\
& +\frac{x}{2} s(x) s\left(x^{3}\right)
\end{aligned}
$$


The sum $\Sigma q^{*}(T) x^{n(T)}$ counts dissimilar carbon lines in steric trees. We can think of a steric tree with a distinguished carbon line as a function from the two ends of the line to the two planted steric trees obtained by severing the line and planting the resulting connected components at the cut ends. The allowable group of permutations on the two ends of the distinguished line is the symmetric group $S_{2}$, and hence has cycle index:

$$
\frac{1}{2} a^{2}+\frac{1}{2} a_{2} \text {. }
$$

The planted trees which may be associated with the two ends of the distinguished line do not include the trivial one, so they are counted by the series $s(x)-1$. By Pólya's Hauptsatz, then,

$$
\sum q^{*}(T) x^{n(T)}=\frac{1}{2}\left[(s(x)-1)^{2}+s\left(x^{2}\right)-1\right],
$$

giving the second term of (6).

Finally, the sum $\Sigma s^{*}(T) x^{n(1)}$ counts those carbon lines in steric trees which join carbon points which are interchanged by some steric automorphism of the tree. Again severing the line to obtain two planted steric trees, we observe that in this case the two planted steric trees must be isomorphic and non-trivial. To count isomorphic pairs of planted steric trees, we simply substitute $x^{2}$ for $x$ to double the carbon point count in the series $s(x)-1$ which enumerates the non-trivial planted steric trees. Thus

$$
\sum s^{*}(T) x^{n(r)}=s\left(x^{2}\right)-1 .
$$

Substituting (8), (9) and (10) into (6), we obtain

$$
\begin{aligned}
f(x)=\frac{1}{4}\left[2 x s(x) s\left(x^{3}\right)+3 s(x)\right. & +2 s\left(x^{2}\right) \\
& \left.+s^{2}\left(x^{2}\right)-s^{2}(x)\right]-1 .
\end{aligned}
$$

Recalling the definition (3) of $q_{n}$, equation (11) gives the recurrence relation

$f_{n}=\frac{1}{4}\left[3 s_{n}+2 s_{n / 2}+q_{(n-1) / 2}-q_{n}+2 \sum_{n=n / 3} s_{k} s_{n-3 k-1}\right]$

for $n \geqslant 1$, along with $f_{0}=0$. From the point of view of computation, (12) is in a very convenient form.

\section{COUNTING ACHIRAL STERIC TREES}

3.1 Achiral planted trees. As noted in the introductory section, $s_{n}=g_{n}+p_{n}$. If we add to the admissible isomorphisms by allowing a steric tree to be mapped to its reverse, we would obtain $g_{n} / 2+p_{n}$ for the number of order $n$. We could then multiply by 2 and subtract $s_{n}$ to obtain $p_{n}$. This is the approach which we shall take. The basic idea is due to Pólya, ${ }^{6}$ and was applied by $\operatorname{Read}^{25}$ to selfcomplementary graphs and diagraphs.

In order to count planted steric trees with respect to steric isomorphisms augmented by reversals, we use the same idea as for (1). However, Pólya's Hauptsatz is not directly applicable to this situation. The admissible group of permutations on the three free vertices of the tetrahedron (representing the three bonds of the carbon atom in the corresponding alkyl radical) now contains all 6 possible permutations. The cycle index of this group, which is the symmetric group $S_{3}$, is

$$
\frac{1}{6} a_{1}{ }^{3}+\frac{1}{2} a_{1} a_{2}+\frac{1}{3} a_{3}
$$

Substitution for the terms $a_{1}{ }^{3}$ and $a_{3}$ is unchanged from (1), as these represent exactly the same rotations as before. However $a_{1} a_{2}$ represents a reflection in a plane, which leaves one vertex (besides the root vertex) fixed and interchanges the the other two. The action of this reversal on a function is not simply to permute the domain elements, but also to reverse each value in its range. This means that for $a_{1}$ in $a_{1} a_{2}$ we must substitute $p(x)$, for if the function is to be fixed under the reversal it must map the fixed vertex to an achiral planted tree. But for $a_{2}$ we can still substitute $s\left(x^{2}\right)$, as it is just a matter of assigning an arbitrary planted steric tree to one vertex and the reverse tree to the other vertex. Thus the analogue of (1) is

$$
\sum_{n=0}^{\infty}\left(p_{n}+g_{n} / 2\right) x^{n}=1+x\left\{\frac{1}{6} s^{3}(x)+\frac{1}{2} p(x) s\left(x^{2}\right)+\frac{1}{3} s\left(x^{3}\right)\right\} .
$$

Together with (1) and our initial observation, (13) implies

$$
p(x)=1+x p(x) s\left(x^{2}\right),
$$

which determines $p(x)$ from $s(x)$ at once. Equating coefficients in (14) gives

$$
p_{n+1}=\sum_{k=n i 2} s_{k} p_{n} 2 k .
$$

With the initial condition $p_{0}=1,(15)$ is easily solved for successive values of $p_{n}$ in terms of the previously obtained sequence $s_{1}$.

3.2. Achiral free trees. To obtain $t(x)$ in terms of $s(x)$ and $p(x)$ we again use the idea of Otter's dissimilarity characteristic equation. For any achiral tree $T$, let $T^{*}$ be the subgraph of $T$ induced by those carbon points which are left invariant by some steric isomorphism of $T$ to its reverse, that is, consisting of those carbon points and the lines in $T$ which join them. It is not hard to see that $T^{*}$ is either empty or else a tree (the proof for the planar case is in Section 4 of Ref. ${ }^{19}$, and can be applied here with little change). Let $p^{\prime \prime}(T)$ be the number of carbon points of $T^{*}$ which are dissimilar under the steric automorphisms of $T$, and let $q^{\prime \prime}(T)$ be the corresponding number of dissimilar carbon lines. Let $s^{*}(T)$ be 0 or 1 , the latter just if $T^{*}$ contains a pair of adjacent points which are sterically similar or if $T^{*}$ is empty.

In case $T^{*}$ is not empty, $T^{*}$ is a subtree of $T$, so the justification for the dissimilarity characteristic equation (5) can be applied to show that

$$
p^{* \prime}(T)-q^{*}(T)+s^{*}(T)=1 .
$$

If, on the other hand, $T^{*}$ is empty, then $p^{\prime \prime}(T)=q^{\prime \prime}(T)=$ 0 while $s^{\prime \prime}(T)=1$. Thus (16) is valid for every achiral tree $T$.

Recall that $n(T)$ is the order of $T$, i.e., the number of carbon points in $T$. We multiply both sides of (16) by $x^{n(T)}$ and sum over all achiral trees to obtain

$$
\begin{aligned}
\sum p^{\prime \prime}(T) x^{n(T)}-\sum q^{\prime \prime}(T) x^{n(T)}+\sum s^{\prime \prime} & (T) x^{n(T)} \\
& =\sum x^{n(T)}
\end{aligned}
$$

The right side of (17) is just $t(x)$. It remains to evaluate the three sums of the left side in terms of $s(x)$ and $p(x)$. 
First, $\Sigma p^{*}(T) x^{n(T)}$ counts dissimilar points in the subtrees $T^{*}$. This is equivalent to counting achiral carbon-rooted steric trees, since every point of $T^{*}$ is a carbon point left fixed by some isomorphism of $T$ to its reverse. Much as in the derivation of (14), we shall count all carbon-rooted steric trees under steric isomorphisms augmented by reversals, double this and subtract the count taken with respect to the steric isomorphisms alone. The difference will be just the achiral carbon-rooted trees.

As in the derivation of (7), we note that carbon-rooted steric trees are equivalent to functions from the vertices of a regular tetrahedron into the set of planted steric trees. If we augment the rotations of the tetrahedron by reflections in its planes of symmetry, corresponding to reversals in the associated tree, we obtain the full symmetric group of 24 permutations on the vertices. This group $S_{4}$ has cycle index

$$
\frac{1}{12} a_{1}{ }^{4}+\frac{1}{4} a_{1}{ }^{2} a_{2}+\frac{1}{8} a_{2}{ }^{2}+\frac{1}{3} a_{1} a_{3}+\frac{1}{4} a_{4}
$$

The terms corresponding to reversals are $a_{1}^{2} a_{2}$ and $a_{4}$. So just as for (13) we must substitute $p(x)$ for $a_{1}, s\left(x^{2}\right)$ for $a_{2}$ and $s\left(x^{4}\right)$ for $a_{4}$ in these terms only, and $s(x)$ for $a_{1}$, $s\left(x^{3}\right)$ for $a_{3}$ in the other two terms. The resulting counting series is

$$
\frac{x}{24} s^{4}(x)+\frac{x}{4} p^{2}(x) s\left(x^{2}\right)+\frac{x}{8} s^{2}\left(x^{2}\right)+\frac{x}{3} s(x) s\left(x^{3}\right)+\frac{x}{4} s\left(x^{4}\right),
$$

after multiplying through by $x$ to include the root point in the count of carbon points. In (18) each enantiomeric pair is counted just once, so by doubling (18) and subtracting the expression (8) for ordinary steric trees we are left with an expression for the achiral ones alone. Thus we obtain the simplified equation

$$
\sum p^{\prime \prime}(T) x^{n(T)}=\frac{x}{2} p^{2}(x) s\left(x^{2}\right)+\frac{x}{2} s\left(x^{4}\right) .
$$

Next, $\Sigma q^{*}(T) x^{n(T)}$ counts dissimilar carbon lines in the subtrees $T^{*}$. Now, cutting a carbon line in $T^{*}$ and planting each of the two resulting components of $T$ at the cut ends results in a pair of non-trivial planted steric trees. Since each endpoint of the original line in $T^{*}$ is left fixed by some steric automorphism of $T$ to its reverse, it is not hard to see that each of the two resulting planted trees is achiral. Conversely, if we form a tree $T$ by joining two non-trivial achiral planted trees at their roots, then suppress this root to just an ordinary point in a new line, then $T$ will be achiral and the new line will be in $T^{*}$. Thus, by analogy with (9), we have

$$
\sum q^{\prime \prime}(T) x^{n(T)}=\frac{1}{2}[p(x)-1]^{2}+\frac{1}{2}\left[\left(p\left(x^{2}\right)-1\right] .\right.
$$

Finally, $\sum s^{*}(T) x^{n(T)}$ can be thought of as counting unordered pairs of non-trivial planted steric trees which are mutually reverse. Indeed, when $T$ is achiral a necessary and sufficient condition for $T^{*}$ to contain an adjacent pair of similar points is that the two branches obtained by severing the center line be isomorphic achiral planted trees. On the other hand, $T^{*}$ is empty for an achiral tree $T$ just if the branches at the center line are a distinct pair of mutually reverse planted steric trees. For the achirality of $T$ implies that $T$ is sterically isomorphic to its reverse, while $T^{*}$ empty implies that no carbon point (and hence no point at all) of $T$ is left fixed by such an isomorphism. Now, ordered pairs of non-trivial mutually reverse planted steric trees are counted by the series $s\left(x^{2}\right)-1$. Dividing by 2 has the effect of counting only $\frac{1}{2}$ for each isomorphic pair, which must in that case be achiral. These are counted by the series $p\left(x^{2}\right)-1$, so the correct expression is

$$
\sum s^{*}(T) x^{n(T)}=\frac{1}{2}\left[s\left(x^{2}\right)-1\right]+\frac{1}{2}\left[p\left(x^{2}\right)-1\right] .
$$

Combining (17), (19), (20) and (21), we find

$$
t(x)=\frac{1}{2}\left[s\left(x^{2}\right)+x s\left(x^{4}\right)+p(x)\right]-1
$$

after using (14) as an aid in simplification. Equating coefficients in (22) gives the initial condition $t_{0}=0$ and the explicit relation

$$
t_{n \cdot 1}=\frac{1}{2}\left(p_{n+1}+s_{(n+1,12}+s_{n / 4}\right)
$$

Tables 2 and 3 present the numbers of various kinds of planted trees and free trees discussed in this paper. The partial numerical values obtained by earlier authors ${ }^{3-5.16-18}$ agree with them.

\section{REFERENCES}

'A. Cayley, Phil. Mag. 67, 444(1874).

${ }^{2}$ A. Cayley, Disch. Chem. bes Ber. 8,1056 (1875).

${ }^{3}$ H. R. Henze and C. M. Blair, J. Am. Chem. Soc. 53, 3042 (1931).

${ }^{4}$ H. R. Henze and C. M. Blair, Ibid. 53, 3077 (1931).

${ }^{5}$ D. Perry, Ibid. 54, 2918 (1932).

'G. Pólya, Acta Math. 68, 145 (1937).

${ }^{2} \mathrm{R}$. Otter, Ann. of Math. 49, 583 (1948)

${ }^{8} \mathrm{~F}$. Harary, Graph Theory. Addison-Wesley, Reading, Mass. (1969).

${ }^{9}$ F. Harary and E. M. Palmer, Grophical Enumeration. Academic Press, New York (1973)

${ }^{10}$ D. H. Rouvray, Roy. Inst. Chem. Rev. 4, 173 (1971); Chem. Brit. 10, 11 (1974); Chem. Soc. Rev. 3, 355 (1974); Endeavour 34, 28 (1975).

'R. C. Read, Graph Theory and Applications (Edited by Y. Alavi et al.). Springer-Verlag, Berlin, 243 (1972).

'2J. H. van't Hoff, Arch. Neer. 9, 445 (1874).

${ }^{13}$ J.-A. LeBel, Bull. Soc. Chim. Fr. [2] 22, 337 (1874).

${ }^{14}$ R. Robinson, Tetrahedron 30, 1477 (1974).

${ }^{15}$ F. G. Riddell and M. J. T. Robinson, Ibid. 30, 2001 (1974).

${ }^{16} \mathrm{C}$. M. Blair and H. R. Henze, J. Am. Chem. Soc. 54, 1098 (1932).

${ }^{17} \mathrm{C}$. M. Blair and H. R. Henze, Ibid. 54, 1538 (1932).

${ }^{18}$ R. C. Read, Chemical Applications of Graph Theory. (Edited by A. T. Balaban). Academic Press, London, to appear.

${ }^{19} \mathrm{~F}$. Harary and R. W. Robinson, J. Reine Angew. Math. to appear.

${ }^{20}$ S. L. Murov and M. Pickering, J. Chem. Educ. 50, 74 (1973); and references quoted.

${ }^{21} \mathrm{H}$. Wynberg and L. A. Hulshot, Tetrahedron 30, 1225 (1974); J. H. Brewster, Ibid, 1807; S. D. Allen and O. Schnepp, J.C.S. Chem. Comm. 904 (1974).

${ }^{22} \mathrm{~F}$. Harary and R. C. Read, Graphs and Combinatorics, (Edited by R. Bari and F. Harary), p. 37. Springer-Verlag, Berlin (1974).

${ }^{23} \mathrm{~W}$. Burnside, Theory of Groups of Finite Order (2nd Edn) Theorem VII, p. 191. Cambridge Univ. Press, London (1911); reprinted by Dover, N.Y., 1955

${ }^{24}$ T. L. Hill, J. Chem. Phys. 11, 294 (1943).

${ }^{25}$ R. C. Read, J. London Math. Soc. 38, 99 (1963). 